\title{
Correspondence
}

Epidemiol. Infect. (2012).

doi: $10.1017 /$ S0950268811002512

First published online 6 December 2011

\section{Spatial epidemiology of leptospirosis in Sri Lanka}

To the Editor

Robertson and colleagues [1] analysed the spatial epidemiology of leptospirosis in Sri Lanka and showed a probable correlation between occurrence of leptospirosis and rainfall patterns in Sri Lanka. They also identified risk clusters for leptospirosis based on the spatial distribution of the reported cases. We appreciate this work which filled several knowledge gaps in the epidemiology of leptospirosis in Sri Lanka. However, we are concerned about some facts in the paper which need further explanation and corrections.

The disease rates in the paper were analysed using routinely reported data. Authors have correctly described that these were not confirmed cases. They further provide evidence to suggest that the reported cases would be valid for this analysis. The supportive evidence [2] provided for validity of reported cases was not actually for the reported cases. We carried out the particular study to validate the WHO proposed surveillance case definition. Although this case definition is recommended for case reporting, the clinical practice and reporting is completely different from this case definition. Since the laboratory diagnosis is not routinely available, reporting is entirely based on clinical judgement. We previously analysed this error in our study conducted during the 2008 epidemic, in Kandy, Kegalle and Matale, districts which showed that only $52 \cdot 6 \%$ of the patients treated for leptospirosis actually had the disease. Furthermore, $46.2 \%$ of the patients who were treated for other conditions were retrospectively confirmed as leptosprosis cases [3]. To show the diversity of the clinical judgement we further analysed these cases by reporting hospitals. It showed that only $28.3 \%$ of cases from Matale were confirmed positive, compared to $58.3 \%$ and $55.0 \%$ in Kandy and Kegalle hospitals, respectively. According to the routinely reported data, Matale had the highest incidence of leptospirosis during the 2008 epidemic, which we proved as not the correct figure. It is clear that Matale had a higher number of cases during the 2008 outbreak. However, fewer than one third of the suspected and reported cases actually had the disease and it was significantly lower than in other areas. The reported data available in the surveillance system seems to be an overestimation of leptospirosis incidence in Matale and we suspect that this had affected the Robertson study in which the authors discussed Matale specifically. To further explain this diversity of clinical judgement, we looked at the cases reported from the three medical units in Kandy hospital. While one unit reported 54 clinically suspected cases during our study period of 4 months, the other two units each reported fewer than 10 suspected cases. This difference is highly unlikely, given that the same number of admission days are allocated to each unit. The most plausible explanation is that clinical suspicion varied widely according to treating physicians. While the 2008 outbreak of leptospirosis undoubtedly existed, exact case load and geographical distribution are questionable due to the lack of point-of-care diagnostic facilities and gross under-/overreporting of cases, based on the treating physician.

We have another major concern about the use of 2002 data for agriculture/paddy field distribution. In 2007, the government launched an island-wide programme ('Api Vavamu, Rata Nagamu'), which made it mandatory to cultivate all abandoned paddy fields. This was seen especially in 2008, where a large number of people who were not traditional farmers got involved in paddy farming activities. This programme would have changed the paddy field distribution in 2002 considerably, and it may be a reason for not showing a significant association with the 2008 outbreak.

We would like to point out some other inaccuracies in the paper: (1) Sri Lanka does have a seasonal 
pattern for rainfall, but being a tropical country, we do not have four seasons as described by the authors. (2) The authors referred to our study published in 2008 [4] as evidence for diversity of serovars during the 2008 outbreak. This report was for the 2002-2003 period, not for the 2008 outbreak. (3) The authors referred to the report on interim analysis of the 2008 outbreak and mentioned that nine serovars were isolated. There has been no published literature on serovar isolation from Sri Lanka recently. The citation in the paper was based on results of the microscopic agglutination test. (4) In the paper, the authors used $\mathrm{MOH}$ areas as the unit of analysis, and $\mathrm{MOH}$ was defined as 'Ministry of Health'. This is incorrect $-\mathrm{MOH}$ areas are 'Medical Officer of Health' areas, which are divisional-level health administrative units in Sri Lanka.

We also were very interested as to why authors reported the 'prevalence' of leptospirosis. Conventionally, we express leptospirosis disease as incidence because it is an acute condition.

\section{Declaration of Interest}

None.

\section{References}

1. Robertson C, Nelson TA, Stephen C. Spatial epidemiology of suspected clinical leptospirosis in Sri Lanka. Epidemiology and Infection. Published online: 7 June 2011. doi:10.1017/S0950268811001014.

2. Dassanayake D, et al. Evaluation of surveillance case definition in the diagnosis of leptospirosis, using the microscopic agglutination test: a validation study. $B M C$ Infectious Disease 2009; 9: 48.

3. Agampodi SB, et al. Leptospirosis outbreak in Sri Lanka in 2008: lessons for assessing the global burden of disease. American Journal of Tropical Medicine and Hygiene 2011; 85: 471-478.

4. Agampodi SB, et al. A preliminary study on prevalent serovars of leptospirosis among patients admitted to teaching hospital, Kandy, Sri Lanka. Indian Journal of Medical Microbiology 2008; 26: 405-406.

S. B. AGAMPODI (on behalf of the authors)

Department of Community Medicine, Faculty of Medicine and Allied Sciences, Rajarata University of Sri Lanka Department of Medicine, School of Medicine, University of California, San Diego

Address for correspondence:

Dr S. B. Agampodi

Division of Infectious Diseases, Department of Medicine, UCSD School of Medicine, 9500 Gilman Drive 0741,
George Palade Laboratories, Room 126,

La Jolla, CA 92093-0741, USA

(Email: sunethagampodi@yahoo.com)

Epidemiol. Infect. (2012).

doi:10.1017/S0950268811002524

First published online 6 December 2011

\section{The authors reply}

We thank Dr Agampodi for his comments on our paper describing recent spatial-temporal patterns in suspected clinical leptospirosis in Sri Lanka, and hope to address some of the concerns raised in the letter. The first main criticism, being the validity of the surveillance data used in this analysis, highlights a general shortcoming of performing epidemiological analysis over large geographical areas in countries with inconsistent surveillance and reporting when timely diagnoses is neither sensitive nor specific. We acknowledged this issue throughout the paper, discussing the possibility of hantavirus or dengue presenting as leptospirosis, not to mention entitling our paper 'suspected clinical leptospirosis' as a way of further highlighting this uncertainty. This of course begs the question, whether it is worth doing analysis of risk for cases with uncertain diagnoses, perhaps due in part to variation in clinical practice. We would argue that this type of analysis is necessary for these data because of the uncertainty associated with such diagnoses. One of the key purposes of surveillance data is to monitor trends in the health status of populations, what labels we attach to these conditions matter less than the fact that the number of people with acute febrile illness was unusually high. So faced with this uncertainty, we looked for correlative risk factors. Geographical risk analysis of surveillance data at the scale done here is by its very nature exploratory and inductive.

In the analysis presented, we detected clusters of cases in space and time, correlated these clusters with risk factors, interpreted patterns in light of the probable mechanisms, and concluded with avenues for future research. To address the specific criticisms raised by Agampodi, the paper [1] which describes its aim to validate the leptospirosis case definition in Sri Lanka using the microscopic agglutination test (MAT), does not report variation in clinical practice as a limitation in that study. It is therefore not unusual that we would use its findings as supportive evidence for doing a geographical risk analysis based on surveillance data, despite our noted warnings about misdiagnosis and clinical uncertainties. We also 